\title{
Historiographie médiévale et moderne en Europe occidentale
}

Jean-Marie Moeglin

\section{OpenEdition}

Journals

Édition électronique

URL : https://journals.openedition.org/ashp/4439

DOI : $10.4000 /$ ashp.4439

ISSN : 1969-6310

Éditeur

Publications de l'École Pratique des Hautes Études

Édition imprimée

Date de publication : 1 septembre 2021

Pagination : 267-280

ISSN : 0766-0677

\section{Référence électronique}

Jean-Marie Moeglin, « Historiographie médiévale et moderne en Europe occidentale », Annuaire de

I'École pratique des hautes études (EPHE), Section des sciences historiques et philologiques [En ligne],

152 | 2021, mis en ligne le 14 juin 2021, consulté le 13 juin 2022. URL : http://journals.openedition.org/ ashp/4439; DOI : https://doi.org/10.4000/ashp.4439 


\title{
HISTORIOGR APHIE MÉDIÉVALE ET MODERNE EN EUROPE OCCIDENTALE
}

\author{
Directeur d'études : M. Jean-Marie MoEgLIN
}

Programme de l'année 2019-2020 : I. Le rituel de la corde au cou. - II. Le viol de la comtesse de Salisbury et la fondation de l'ordre de la Jarretière. - III. Questions d'historiographie médiévale et moderne.

Dans une année quelque peu perturbée par la pandémie, la conférence s'est avant tout préoccupée de clarifier un problème érudit concernant l'histoire du viol de la comtesse de Salisbury. L'on sait qu'un étonnant récit du chroniqueur liégeois Jean Le Bel rapporte comment le roi d'Angleterre Édouard III a été saisi lors d'une expédition contre les Écossais d'un amour fou pour la dame de Salisbury, Alice, épouse d'un de ses plus proches barons, William Montague, $1^{\text {er }}$ earl de Salisbury. D'abord éconduit par la comtesse, il revient, après le départ de son époux pour la guerre de Bretagne, au château où elle séjourne, et il la viole sauvagement. Le comte prévenu à son retour par son épouse dénonce le roi publiquement devant la cour avant de s'exiler et de mourir au siège d'Algésiras contre les Musulmans.

Il s'agit en réalité d'une fiction qui ne correspond pas à la réalité historique comme le montrent de nombreuses invraisemblances historiques. L'hypothèse, généralement retenue, d'une rumeur lancée par les ennemis d'Édouard et reprise par mégarde par Jean Le Bel, un historien parfaitement maître de sa prose, est également exclue. Il s'agit bien d'une invention volontaire de Jean Le Bel, admirateur du «noble roi Édouard » mais déçu et amer que ce dernier, qui se présentait pourtant comme le nouveau roi Arthur, ait renoncé, après sa victoire de Poitiers en 1356, à conquérir la couronne de France usurpée par Philippe VI, le tyran que Jean Le Bel déteste.

Cette histoire terrible n'est racontée que par Jean Le Bel dans sa chronique écrite au cours des années 1350 ; elle est toutefois reprise vers 1370 , sous une forme très succincte, par l'auteur de la Chronique normande du XIV ${ }^{e}$ siècle dont le texte sera recopié par quelques autres œuvres. Il a été soutenu que le récit de la Chronique normande serait indépendant de celui de Jean Le Bel et il importe d'examiner de près cette hypothèse afin de montrer qu'elle ne peut être retenue.

Il existe en effet plusieurs textes au $\mathrm{XIV}^{\mathrm{e}}$-début $\mathrm{XV}^{\mathrm{e}}$ siècle qui rapportent l'histoire du viol de la comtesse de Salisbury. Le premier problème à résoudre est de savoir s'ils sont indépendants les uns des autres. L'on dispose en effet non seulement du récit de Jean Le Bel mais aussi des mentions contenues dans la Chronique normande, la Chronique normande abrégée, la continuation de la Chronique de Flandre après 1342 et la Chronographia regum Francorum. Ces quatre dernières chroniques donnent exactement le même texte (les trois premières en français, la quatrième en latin). Cela s'explique par le fait que la continuation de la Chronique de Flandre et la Chronographia ont toutes deux recopié la Chronique normande abrégée qui a elle-même recopié la Chronique normande (on pourrait éventuellement avancer que la Chronique normande a recopié la Chronique normande abrégée mais c'est peu probable). 
La comparaison du récit du viol de la comtesse de Salisbury dans ces différentes chroniques le montre :

- La Chronique normande du XIV siècle

Après ce que Anglois furent entrez en mer, le roy Edouart d'Angleterre viola par force la femme du conte de Salbery, qui pour lui èstoit alé en la guerre à son commandement. $[\ldots]$

En l'ost du duc. Jehan, estoit Olliviers de Clicon, Gaudeffroy de Harrecourt et pluseurs autres chevaliers devant nommez, qui par trahison s'estoient aliez au conte de Montfort, et aussi se alierent.il au roy Edouart par dons et par promesses. Et fut faite une lettre d'aliance, où li pluseurs mistrent leurs seaulz, et celle lettre garda le conte de Salbery de par le roy Edouart.

[...] Après ce que le roy de France et le roy d'Angleterre furent retraiz en leurs pais, le conte de Salbery sceut par sa femme que elle avoit esté par force violée du roy d'Angleterre. Le conte fist son atrait et manda ses amis et s'en ala à la court du roy Edouart devant les pars et se dessaisi de sa terre par tele manière que sa femme y prendroit son douaire sa vie durant. Dont se parti le conte de Salbery de la court et envoya deffier le roy Edouart, et passa la mer et vint au roy Phelippe et lui bailla les lettres de l'aliance, que Oliviers de Clicon et Godeffroy de Harecourt avoient fait au roy Edouart. Et moût tost après se parti le conte de la court au roy Phelippe, et puis ce temps ne fut veu en France ne en Angleterre.

Adonc fu pris Olliviérs de Clicon et le fist le rois décoller à Paris sur I escaffault et fist le corps pendre à Monfaucon ...

(A. et E. Molinier, Chronique normande du XIVe siècle, Paris, 1882 [SHF], p. 53-57.)

- Continuation de la Chronique de Flandre (= Chronique normande abrégée)

Après ce que li Englès furent entret en mer, li rois Edouwars viola par forche le contesse de Salbrin, femme du conte de Salbrin, qui pour lui estoit alés en la guerre par son command. [...]

Après ce que li doy roy se furent retrait en leurs pays, li contes de Salbrin seut par se femme meisme que par forche elle avoit esté violée du roy Edouart. Dont fist li contes son atrait, et manda ses amis, et s'en ala a la court devant ses pers et se dessaisi de se terre et en ahireta une fille qu'il avoit, par tant que se femme y prendroit sen douaire toute se vie. Puis se parti li contes de le court Edouwart, et s'en vint par dechà, et envoya deffyer Edouwart, et vint au roy Phelippe et li bailla le lettre de l'alianche que Oliviers de Clichon et Godefroys de Harcourt avoyent fait au roy Edouwart; et moult tost après se parti li contes de le court du roy Phelippe, et puis ce temps ne fu veus en Franche, ne en Engleterre.

Àdont fu pris Oliviers de Clichon, et le fist li rois décoler à Paris sur un escaffault, et fist $\mathrm{y}$ corps pendre à Monfaucon...

(Kervyn de Lettenhove, Istore et croniques de Flandres, t. II, Bruxelles, 1880, p. 6, 9.)

\section{- Chronographia Regum Francorum}

Cum ergo hec fierent, rex Anglie violavit uxorem predicti comitis Saresberiensis.

$[\ldots]$

Post regressum dictorum regum ad propria, comitissa Salesberiensis conquesta est marito suo de sui violatione a rege Anglie; qui de hoc mestus et tristis ultramodum, convocavit aimicos suos atque cum eis abiens ad curiarn regis Anglie, ubi coram eo, presentibus paribus regni, dissaisiit se de tota terra sua et ea hereditavit filiam suam quia masculinum heredem non habebat, sic quod uxor ejus haberet doagium quamdiu viveret. 
Deinde recedens de curia sua, misit qui pro eo diffideret regem Edowardum atque transmeavit in Franciam ad regem Philippum et tradidit ei litteras de confederatione dictorum Oliveri de Cliçonio et Godefridi de Haricuria cum rege Anglie. Et modicum post, comes recessit de Francia, nec postea a quoquam visus est.

Captus est itaque Oliverus de Cliçonio, atque jussu regis Philippi decapitatus est in quodam spectaculo Parisius; corpus autem ipsius suspensum est in patibulo quod Monsfalconis dicitur, prope Parisius...

(H. Moranvillé éd., Chronographia Regum Francorum, t. II, Paris, 1893, p. 197, 204205.)

Ces textes sont rigoureusement identiques et leurs auteurs se sont donc forcément recopiés.

Pour déterminer qui a recopié qui, la logique est de prendre l'ordre chronologique de rédaction de ces chroniques et donc de conclure que la Chronique normande, en tant que texte le plus ancien, est la source des autres.

Henri Moranvillé a toutefois, dans l'introduction de son édition de la Chronographia pour la Société d'histoire de France, semé le doute en émettant l'idée que la Chronographia était la copie d'un texte antérieur qui aurait lui-même été recopié notamment par la Chronique normande et la Chronique de Flandre. Sa théorie a été immédiatement balayée aussi bien par Jules Viard, «La Chronique de Jean le Bel et la Chronographia regum Francorum », BEC, 66 [1905], p. 540-546) que par Henri Pirenne ("L'Ancienne chronique de Flandre et la Chronographia regum Francorum », Compte rendu des séances de la commission royale d'histoire, $2^{\mathrm{e}}$ série, 8 [1898], p. 199-208). Cela n'a malgré tout pas suffi pour éradiquer cette théorie et c'est elle qui a guidé Antonia Gransden dans son article de 1971 lorsqu'elle a voulu montrer que Jean Le Bel, dans l'affaire du viol de la comtesse de Salisbury, s'était laissé tromper par la propagande française. Je redonne donc à nouveaux frais cette démonstration.

La Chronographia regum Francorum est un texte conservé dans un unique manuscrit, de la Burgerbibliothek de Berne, $\mathrm{n}^{\circ} 73$ qui pourrait dater du milieu du Xv $\mathrm{Xv}^{\mathrm{e}}$ siècle. Il s'agit d'un recueil des hauts faits des rois de France, règne par règne, avec au commencement de chaque règne un dessin représentant le roi en question. Elle regroupe toutefois deux parties bien distinctes; en effet le prologue de la chronique, repris à la Chronique des rois de France de Guillaume de Nangis, annonce une histoire des rois de France qui va simplement jusqu'à saint Louis. Ensuite vient une seconde partie, publiée par Henri Moranvillé, qui est introduite par les mots incipit cronographia regum Francorum subsequencium et quorundam aliorum et primo de Philippo quarto [= Philippe III]. Cette division apparente en deux parties n'empêche pas l'auteur de considérer son œuvre comme un tout; on peut néanmoins, par commodité, dire que la chronographia regum Francorum est la seconde partie du manuscrit. Elle va jusqu'en 1405.

La chronique se termine avec l'annonce de la paix (fourrée) conclue entre les oncles du roi et le frère du roi, qui devait régler un violent conflit entre le duc de Bourgogne et le duc d'Orléans, le 16 octobre 1405, et par l'annonce du retour à Paris, le 23 octobre, de la reine Isabeau qui s'installe à l'hôtel Saint-Paul, et du duc d'Orléans, qui prend logis dans une résidence située non loin : qua intentione vero quisque, quod 
factum est, fecerit, dijudicare poterit qui corda novit... écrit l'auteur, et le texte prend fin avec le célèbre sermon Vivat rex prononcé par Jean Gerson le 7 novembre 1405.

On peut donc penser que la chronique a été achevée à la fin de l'année 1405 alors qu'une paix a été en principe conclue entre les ducs de Bourgogne et d'Orléans après l'échec de l'offensive du duc de Bourgogne. Henri Moranvillé a pourtant daté la Chronographia de la période 1415-1422 (ou 1429); cette datation, généralement retenue par l'historiographie, ne résiste pourtant pas à un examen sérieux. La date de 1415 est justifiée ainsi par Moranvillé : "d'une part, il a écrit après 1415, parce que au cours d'une de ses digressions généalogiques, il note que Charles d'Albret fut connétable de France », mais la note que Moranvillé place à cet endroit de son édition («créé connétable en 1415 ») n'est pas exacte : Charles d'Albret l'était devenu après la mort de Louis de Sancerre en février 1403; destitué par les Bourguignons en septembre 1411, il retrouve son épée de connétable en septembre 1413. Le passage du compilateur signifie donc simplement qu'il rapportait en ou après 1403 les faits de l'année 1365. Quant à la date de 1429, elle est justifiée ainsi : "d'autre part, il a rédigé sa compilation avant 1429, époque de la fin du Grand Schisme, puisqu'il dit, à la date de 1377 : anno quoque a Nativitate Domini M CCC LXXVII vicesimum quartum scisma fuit et cepit, quod adhuc, Domino permittente, durat. Donc c'est entre 1415 et 1429 que notre compilateur a coordonné son œuvre » (III, p. XLVII). Cela signifie en réalité que le compilateur a écrit son texte avant 1417 (élection de Martin V), probablement même avant le concile de Pise en 1408 qui avait créé un troisième pape (car il évoque plus loin le conflit non réglé entre deux papes), et cela n'exclut en aucune façon, bien au contraire, qu'il l'ait écrit en 1405-1406, au moment où les efforts pour résoudre le schisme étaient aussi fébriles que vains.

On peut donc rejeter sans hésitation une datation « entre 1415 et 1429, peut-être même avant la mort de Charles VI ». Ce texte a été écrit entre 1405 et 1417, probablement dès 1405-1406.

Le point essentiel est cependant de déterminer comment cette compilation a été rédigée.

Moranvillé constate que le texte de la Chronographia fait apparaître d'importantes similitudes avec plusieurs autres textes : la Chronique de Flandre, la Chronique normande, la Chronique normande abrégée, ainsi que les Grandes chroniques de France. Le fait est exact mais ne supporte pas l'explication qu'il en donne : la Chronographia serait la copie quasi parfaite d'un texte latin qui aurait servi de source aux trois autres textes qui l'auraient utilisée indépendamment l'une de l'autre.

En fait, la Chronographia a utilisé comme sources la Chronique de Flandre et la Chronique normande abrégée (qui elle-même avait recopié la Chronique normande) et elle a traduit leur texte en latin. Quant aux Grandes Chroniques de France, elles ont utilisé comme source la Chronique de Flandre (Cf. I. Guyot-Bachy, J.-M. Moeglin, «Comment ont été continuées les Grandes Chroniques de France dans la première moitié du XIV ${ }^{\mathrm{e}}$ siècle », BEC, 163 (2006), p. 385-433).

Plusieurs arguments permettent de démontrer l'utilisation par l'auteur de la Chronographia de la Chronique de Flandre et de la Chronique normande, ou plus exactement de sa variante qu'est la Chronique normande abrégée : 
1. S'il y avait un même texte commun sélectivement utilisé par la Chronique de Flandre et par la Chronique normande abrégée, on devrait parfois retrouver des passages identiques entre l'une et l'autre chronique; il n'y a en effet aucune raison que les deux auteurs aient choisi systématiquement dans leur source commune des passages que l'autre n'a pas repris; or il n'y a quasiment aucun recoupement entre les deux chroniques avant 1342 et la continuation de la Chronique de Flandre à l'aide de la Chronique normande abrégée.

2. L'auteur de la Chronographia ne cache pas qu'il a utilisé plusieurs sources; il a en effet un tic d'écriture classique des compilateurs quand ils passent d'une source à une autre. L'utilisation de sources différentes est souvent annoncée par un quidam dicunt, ou quidam autem dicunt, ferunt autem quidam... En voici un exemple pris dans Chronographia, I, p. 73-74: le compilateur rapporte en suivant la Chronique normande et une source non identifiée les problèmes des Anglais à Gand en 1298; puis il passe à une copie de la Chronique de Flandre concernant le même fait et il l'introduit par un dicunt vero quidam, quod cum Edowardus, rex Anglie et Guido, comes Flandrie, cum multis principibus Almannie essent in Gandavo..., et plus loin, continuant le récit de la Chronique de Flandre (mais celle-ci est passé à un autre événement), il place la clausule dicunt eciam quod...; toutefois, il ne s'est pas aperçu qu'il a déjà raconté une page auparavant à partir de la Chronique normande, donc de manière différente, ce même événement à savoir les rixes entre Gantois et Anglais / Gallois!

3. Ceci renvoie au troisième argument; il arrive que l'auteur de la Chronographia ait été capable de combiner de manière harmonieuse les deux sources, la Chronique de Flandre et la Chronique normande abrégée, racontant de manière différente les mêmes événements; mais parfois il n'a pas fait attention et se contredit d'un passage (tiré de la première source) à un autre passage (tiré de la seconde source). J'en donne un exemple : la Chronographia avait rapporté (I, p. 42-43) en suivant la Chronique de Flandre comment la fille du comte de Flandre, fiancée du fils du roi d'Angleterre, était morte de désespoir au Louvre. Mais plus loin (I, p. 53-54), l'auteur de la Chronographia insère dans sa copie de la Chronique de Flandre un assez long emprunt à la Chronique normande sur les démêlés du comte de Flandre et du roi de France :

... Et etiam cum hoc, juravit predictus Guido comes, quod amodo nullam confederacem faceret cum Edowardo rege Anglie neque cum aliis quibuscumque inimicis regni Francie [fin provisoire de la copie de la Chronique de Flandre et transition vers la Chronique normande]. Eo igitur in terram suam reverso, multas requestas per plures principes et prelatos fecit Philippo regi Francorum quatinus redderet ei filiam suam Philippam, et si quid forefecerit, paratus erat emendare secundum judicium parium Francie. Sed idem rex, habito consilio, nullum responsum dedit. Tunc comes misit procuratores suos Romam ad papam Bonifacium VIIIum, et appellaverunt contra regem Philippum...

Moranvillé écrit dans une note (I, p. 54, n. 1) : «si les anciennes chroniques de Flandre ont commis la grave erreur de dire qu'à ce moment la fille du comte de Flandre était déjà morte, on notera que notre chronique, plus exacte, ne dit rien de semblable ». En réalité, la Chronographia avait elle aussi rapporté la mort de la 
fille du comte de Flandre quand elle suivait plus haut le récit de la Chronique de Flandre, mais, du fait de sa copie de la Chronique normande, elle ressuscite ce pourquoi l'auteur arrête sa copie de la Chronique de Flandre lorsque celle-ci continuait par : « Mais, si tost qu'il fust revenus en Flandres, et il vit que sa fille estoit morte, si manda au roy d'Engleterre que, pour l'amour de Dieu, il luy voulsist aidier à vengier l'outrage que li rois de France luy avoit fait, et li rois d'Engleterre le luy accorda, et fu li aliance jurée et confermée entre eulx » (Kervyn, I, p. 207).

L'auteur de la Chronographia a donc utilisé simultanément la Chronique de Flandre et la Chronique normande abrégée et puis, à partir de 1342, lorsque la Chronique de Flandre se termine dans sa partie originale et qu'un continuateur utilise la Chronique normande abrégée pour poursuivre le récit, c'est désormais cette dernière qui devient la base de son texte.

Au demeurant, l'auteur de la Chronographia a aussi utilisé d'autres sources identifiées (Chronique de Cuvelier, Vœux du Héron, chronique de Jean Le Bel pour quelques courts passages...) ou non identifiées, tandis que certains passages (notamment les excursus généalogiques) devraient lui appartenir en propre.

L'on peut par ailleurs avoir des doutes sérieux sur le fait, soutenu par Moranvillé, que la Chronographia aurait été écrite à Saint-Denis; en effet, elle n'utilise pas comme sources les textes attendus d'un auteur écrivant dans ce monastère (les continuations de Guillaume de Nangis ainsi que Richard Lescot), mais bien plutôt des textes qui renvoient vers le nord du royaume de France (Chronique de Flandre ou Chronique normande abrégée), et pourrait bien y avoir été écrite.

Il ne reste par conséquent que Jean Le Bel et la Chronique normande (avec ses dérivés) comme sources présentant un récit du viol de la comtesse de Salisbury.

S'agit-il de deux traditions indépendantes? Si c'était le cas, l'argument d'Antonia Gransden, sous une forme un peu modifiée, resterait défendable : une rumeur courait que deux auteurs ont recueillie indépendamment l'un de l'autre.

En réalité, l'on peut montrer de manière quasi sûre que le récit de la Chronique normande a sa source directe ou indirecte dans la chronique de Jean Le Bel.

Jean Le Bel et l'auteur de la Chronique normande rapportent en bonne partie les mêmes événements, mais de manière extrêmement différente. Deux passages néanmoins font apparaître une claire dépendance de l'auteur de la Chronique normande vis-à-vis de Jean Le Bel qui lui est un peu antérieur.

L'un est le célèbre récit du sacrifice des bourgeois de Calais pour lequel la Chronique normande est la seule source qui donne un récit fidèle à celui de Jean Le Bel.

L'autre est celui du viol de la comtesse de Salisbury. Or, pour ce dernier, la dépendance s'étend plus largement à tout le récit du (prétendu) voyage en Angleterre au début 1342 de la comtesse de Montfort pour chercher des secours anglais, suivi de son retour en Bretagne en compagnie de Robert d'Artois, du comte de Salisbury et d'autres comtes.

L'ensemble du passage montre en effet de troublantes similitudes entre le récit de Jean Le Bel et celui de la Chronique normande, à commencer par le voyage, en réalité fictif, de la comtesse de Montfort en Angleterre, mentionné uniquement par Jean Le Bel et la Chronique normande; celle-ci, en fait, comme l'avait déjà montré l'Histoire de Bretagne d'Arthur Le Moyne de La Borderie (Histoire de Bretagne, t. III, 
Rennes, Paris, 1899, p. 447; cf. à présent Erika Graham-Goering, Michael Jones, Bertrand Yeurc'h, avec la collaboration de Philippe Charon, Aux origines de la guerre de Succession de Bretagne. Documents (1341-1342), Rennes, 2019, notamment p. 55 et 255-256), n'a pas quitté la Bretagne et les négociations avec le roi d'Angleterre se sont faites par le biais d'intermédiaires effectivement attestés en Angleterre à cette date.

Si l'on va plus loin dans la comparaison, l'on constate que le récit du voyage de la comtesse de Montfort chez Édouard III et des événements qui s'ensuivent en Bretagne chez Jean Le Bel et dans la Chronique normande montre, sans même tenir compte de l'affaire Salisbury, une suite de connexions entre deux chroniques qui sont d'ordinaire clairement indépendantes :

— en italiques : les similitudes entre les deux textes;

— en gras : les passages où les deux textes se contredisent;

— souligné : l'affaire Salisbury proprement dite.

\begin{tabular}{|c|c|}
\hline Chronique de Jean Le Bel & Chronique normande \\
\hline JLB, I, p. 271 & CN, p. 53-57 \\
\hline $\begin{array}{l}\text { Si se partirent les seigneurs et retournèrent à } \\
\text { Paris, et livrerent ledit conte de Montfort au } \\
\text { roy qui en eut moult grande joye, et le fist } \\
\text { mettre en prison au Louvre à Paris, où il } \\
\text { moru comme on m'a dit; se je mesprens, si } \\
\text { me soit pardonné. }\end{array}$ & $\begin{array}{l}\text {.. [Jean de Montfort] se rendi au duc } \\
\text { Jehan, sauve sa vie, et firent ceulz de Nantes } \\
\text { hommaige à Charles de Blois et tout le pais } \\
\text { de Bretaigne Galot, et rendi Pappellons de } \\
\text { Saint Gilé à Charles de Blois le chastel de } \\
\text { Saint Albin de Cormier et s'accorda à lui. }\end{array}$ \\
\hline $\begin{array}{l}\text { Or vueil je retourner à madame la contesse } \\
\text { de Montfort, laquelle avoit cuer de lyon. } \\
\text { Elle estoit à Rennes quant elle entendi que } \\
\text { le conte son mary estoit pris; s'elle en fut } \\
\text { moult et moult dolente ce n'est pas merveille, } \\
\text { car elle pensa mielx qu'on le deust mettre } \\
\text { à mort que en prison, et combien qu'elle } \\
\text { eust grand doeul au cuer, si ne fist elle pas } \\
\text { comme femme, mais comme homme de } \\
\text { grand courage, en reconfortant ses amis et } \\
\text { ses souldoiers, et leur monstroit ung petit filz } \\
\text { qu'elle avoit, et leur disoit... } \\
\text { JLB, II, p. 7-12 } \\
\text { Le jour de Pasques vint, que tous les seigneurs } \\
\text { et communes des bonnes villes d'Angleterre } \\
\text { furent assemblez à Erwick. Là parlementa } \\
\text { on de pluseurs besongnes. Je ne sçay pas } \\
\text { bonnement à quel propos n'en quel terme le } \\
\text { parlement fina, car cil qui le me conta n'estoit } \\
\text { pas du secret conseil des seigneurs, mais tant } \\
\text { en sçay je, que le roy d'Angleterre pas n'ala } \\
\text { à celle foys en Escoce. } \\
\text { Je ne sçay pourquoy il le laissa, mais en ces } \\
\text { entrefaictes là vint la vaillant contesse de } \\
\text { Montfort pour requerre secours, et exposa }\end{array}$ & $\begin{array}{l}\text { Dont se parti l'ost des François et mena le } \\
\text { duc Jehan le conte de Montfort prisonnier à } \\
\text { Paris au roy Phelippe, qui le fist emprisonner } \\
\text { au Louvre. } \\
\text { Quant la contesse seut que son baron estoit } \\
\text { en prison et que il ne pot mercy trouver au } \\
\text { roy Phelippe, elle fist porter le trésor, qui } \\
\text { avoit esté au duc Jehan de Bretaigne, au } \\
\text { chastel de Brest, qui moût estoit fort, et le } \\
\text { gardoit Tanguy du Chastel. Tant fist la dame } \\
\text { que elle assembla pluseurs chevaliers et } \\
\text { soudoiers, et alerent de par elle assaillir l'ille } \\
\text { de Gurende et la conquistrent, et se rendirent } \\
\text { ceulz de l'ille à la contesse, et les conduisoit } \\
\text { Gieuffroy de Malatrait, Fouqués de Lavai, } \\
\text { Henry de Champignay, Olivier Rigaut, Denis } \\
\text { du Plessis et pluseurs autre, et alerent assaillir } \\
\text { la ville de Redon et la prindrent par assaut. } \\
\text { Quant Charles de Blois sceut que la comtesse } \\
\text { s'efforçoit de guerrier, il assembla ses } \\
\text { hommes et remanda secours en France. Et le } \\
\text { duc de Normandie lui envoia XII hommes } \\
\text { d'armes, et les conduisent Le Galois de la } \\
\text { Baume, Robert Bertran et Miles de Noiers. } \\
\text { Quant la contesse seut que Charles s'efforçoit } \\
\text { de venir contre ses gens, elle les fist rétraire }\end{array}$ \\
\hline
\end{tabular}




\begin{tabular}{|c|c|}
\hline Chronique de Jean Le Bel & Chronique normande \\
\hline $\begin{array}{l}\text { au roy le grand meschief de son mary } \\
\text { qui estoit en prison pour tant qu'il avoit } \\
\text { relevé de luy la duchié de Bretaigne, et } \\
\text { comment messire Charles de Bloys avoit } \\
\text { pris et reconquis grande partie de villes et } \\
\text { fortresses de Bretaigne, et estoit en doubte } \\
\text { qu'il ne conquist le remanant, car grande } \\
\text { poissance avoit. Le noble roy fist à la dame } \\
\text { grand feste et grand honnour, et aussy firent } \\
\text { tous les aultres seigneurs pour tant qu'elle se } \\
\text { deffendoit si vassaument et pourchassoit la } \\
\text { besongne de son mary. Si luy dit le noble roy, } \\
\text { par le conseil de messire R[obert] d'Artois, } \\
\text { et luy promit que ainchois que les trêves } \\
\text { fussent faillies, il y envoyeroit tel secours } \\
\text { que elle seroit souverainement vengée de ses } \\
\text { anemis, voire et deust il laisser toutes aultres } \\
\text { besongnes. Et prya tantost le noble roy, } \\
\text { sans delay, audit messire R[obert] d'Artoys, } \\
\text { au conte de Salbry, au conte de Suffort, au } \\
\text { conte de Pennebroch, au conte de Renfort, } \\
\text { au baron de Staffort et à mains aultres } \\
\text { seigneurs qu'ilz prissent de souldoiers tant } \\
\text { qu'ilz eussent jusques à IIII' armeures defer, } \\
\text { X mille hommes à pyé et autant d'archiers, } \\
\text { et s'apareillassent d'entrer en mer, et } \\
\text { n'espargnassent riens tant qu'ilz eussent } \\
\text { remis la vaillant contesse en son héritage } \\
\text { malgré tous ses anemis, et encores, se il le } \\
\text { faloit, il iroit celle part à toute sa poissance. }\end{array}$ & $\begin{array}{l}\text { en leurs fors et s'en ala par mer au roy } \\
\text { Edouart, et lui dist comment son baron estoit } \\
\text { prisonnier, et si tenoit Charles de Blois la } \\
\text { duchié de Bretaigne par la force du duc de } \\
\text { Normendie. Adont requist la dame grant } \\
\text { secours au roy Edouàrt, et il s'i accorda, pour } \\
\text { ce que son baron lui àvoit fait hommaige de la } \\
\text { duchié de Bretaigne. Lors assembla XL mile } \\
\text { hommes, et les conduisoit Robert d'Artois, le } \\
\text { conte de Salbery, le conte de Suffot, le conte } \\
\text { de Beaufort, le baron de Stanffort. Ceulz } \\
\text { entrerent en mer et leurs gens et ramenèrent } \\
\text { la contesse de Montfort et Jehan son filz, que } \\
\text { elle avoit menez avec elle. }\end{array}$ \\
\hline
\end{tabular}




\begin{tabular}{|c|c|}
\hline Chronique de Jean Le Bel & Chronique normande \\
\hline $\begin{array}{l}\text { messire Robert d'Artois et sa compaignie } \\
\text { eurent grandement à faire ou pays de } \\
\text { Bretaigne, et ainchoys qu'ilz y venissent, car } \\
\text { messire Loys d'Espaigne, messire Germain } \\
\text { Charles et messire Otton Doriie sceurent leur } \\
\text { venue, si assemblèrent grande compaignie, } \\
\text { et d'Espaignolz, et de Jennevois sur mer. Si } \\
\text { leur coururent sus par nuit et gaagnerrent } \\
\text { III ou IIII de leurs vasseaulx chargez de } \\
\text { pourveances et tuèrent grand foison de leurs } \\
\text { gens; aussy perdirent des leurs. } \\
\text { Je ne sçay pas comment ilz se départirent, } \\
\text { maiz je sçay bien qu'ilz prinrent port en } \\
\text { Bretaigne assez prez de la cité de Venues, } \\
\text { et l'assiegerrent à II costez et se partirent } \\
\text { en II ostz, car les trêves estoient ja faillies; } \\
\text { mais ilz trouverrent la cité bien garnie de } \\
\text { pourveance et de bonnes gens d'armes. } \\
\text { Dedens estoit l'evesque de la cité et messire } \\
\text { Olivier de Glichon, à grand foison de gens } \\
\text { de son lignage, car il estoit seigneur d'une } \\
\text { partie de la cité, et y estoit messire H[ervé] } \\
\text { de Lyon, cappitaine de par messire Charles } \\
\text { de Bloys, qui grandement deffendirent la } \\
\text { cité, ety avint de belles aventures et grandes } \\
\text { proesses d'ung costé et d'aultre que je ne } \\
\text { sçavroye pas raconter ne dire au vray; si } \\
\text { vault mielx que je m'en taise. Mais bien } \\
\text { sçay que ces seigneurs d'Angleterre seirent } \\
\text { longuement devant la cité ainchois qu'ilz } \\
\text { la peussent avoir, et roberrent et gasterrent } \\
\text { entièrement le pays, et retournoient toudis } \\
\text { bien chargiez à leurs logis. } \\
\text { Au darrain, je ne sçay pas certainement } \\
\text { comment il en avint, mais je ay ouy dire } \\
\text { que la cité fut gaagnie par force, par le } \\
\text { consentement de messire Robert d'Artoys et } \\
\text { de messire Olivier de Clichon, ainsy que la } \\
\text { commune voix en couroit. Si fut toute courue, } \\
\text { pillée et gastée, et toutes les gens chassées } \\
\text { hors; et puis tantost aprez les gens du pays se } \\
\text { rassemblèrent avecques ceulx de la cité par le } \\
\text { pourchas de messire Henry de Lyon et dudit } \\
\text { messire Olivier de Glichon, jasoit qu'on } \\
\text { luy meist sus le contraire, et regaagnerrent } \\
\text { ladite cité sur les Angles et y en tuèrent et } \\
\text { prirent grand foison. }\end{array}$ & $\begin{array}{l}\text { Là estoit Loys d'Espaigne et Otton Doire } \\
\text { pour garder le pais à tout X mil Genevois } \\
\text { et pour aler en mer sur les ennemis Charles } \\
\text { de Blois. Et bien sceurent cil la venue des } \\
\text { Anglois. Dont monta une partie de leurs gens } \\
\text { en galies en mer, et l'autre partie demoura à } \\
\text { terre sur le port. } \\
\text { Quant Anglois cuiderent arriver, Genevois } \\
\text { défendirent fort la descente. La ot grande } \\
\text { bataille, et entretant que les Anglois enten- } \\
\text { doient à gaigner terre, Loys d'Espaigne et } \\
\text { Otton Doire les assaillirent par mer à bien VI } \\
\text { mil Genevois et François. Là, ot bien mort III } \\
\text { mil Anglois et le baron de Stanfort, et convint } \\
\text { aux Anglois retraire en mer, et àlerent arriver } \\
\text { au port de Vennes et assegierent la cité. } \\
\text { A Vennes estoit Oliviers de Clicon, qui estoit } \\
\text { d'une partie de la cité, et estoit cil chevalier } \\
\text { et pluseurs autres aliez, au conte de Mont- } \\
\text { fort secreement et avoient fait hommaige à } \\
\text { Charle de Blois par trahison. } \\
\text { A Vennes estoit Hervi de Lion, qui ce jour } \\
\text { yssi de la cité par la porte de l'eau à tout } \\
\text { VII' hommes de commun, et se comba- } \\
\text { tirent aux Anglois et en tuèrent bien IIII', et } \\
\text { recoustrent une grant proye que il avoient forée } \\
\text { et par force la mistrent en la cité. Une autre } \\
\text { fois yssirent les gens de Vennes, bien xii cens } \\
\text { par le commandement Olivier de Clicon, et } \\
\text { furent trahiz, car ainsi que ilz se combatoient, } \\
\text { Robert d'Artois mena une partie des Anglois } \\
\text { vers la porte de la cité et la conquistrent. } \\
\text { Quant les Bretons, qui estoient sailliz hors, } \\
\text { virent que ilz ne peürent entrer en leur ville, } \\
\text { ilz se retrairent en une forte place vers la cité } \\
\text { et mandèrent secours à Beauves sur mer et } \\
\text { ailleurs tant que l'endemain, heure de prime, } \\
\text { ilz assemblerent bien XX mils hommes et } \\
\text { alerent combatre aux Anglois qui estoient sur } \\
\text { les champs. A celle bataille perdirent Anglois } \\
\text { terre. Quant Robert d'Artois et sa gent, qui } \\
\text { estoient dedens la cité virent ce, ilz 1'yssirent } \\
\text { hors et laissierent dedens v cens Anglois. } \\
\text { Mais les Bretons, qui-en la cité ëstoient, et les } \\
\text { femmes mesmes les assaillirent et occirent } \\
\text { mout de Anglois. }\end{array}$ \\
\hline
\end{tabular}




\begin{tabular}{|c|c|}
\hline Chronique de Jean Le Bel & Chronique normande \\
\hline $\begin{array}{l}\text { Et y fut ledit messire Robert d'Artois } \\
\text { grandement navré, tellement qu'il le convint } \\
\text { reporter en Angleterre ou tantost morut, dont } \\
\text { ce fut grand dommage. }\end{array}$ & $\begin{array}{l}\text { La bataille fut grande, où Robert d'Artois } \\
\text { assembla contre François, Genevois et Bre- } \\
\text { tons. Là furent Anglois reculez et cuida Robert } \\
\text { d'Artois rentrer en la cité, mais il ne pot, car } \\
\text { ses hommes estoient occis et le commun ravoit } \\
\text { gaingnée la ville. Et fut ycellui Robert navrez } \\
\text { moût cruelment en la bataille que onques } \\
\text { puis ce jour ne porta armes, ains mourut } \\
\text { dedens brieftemps après. Et nomment aucuns } \\
\text { celle bataille là bataille de Morles. Ceulz de } \\
\text { Vennes et leurs aidans rentrerent en la cité et } \\
\text { les autres Bretons, François et Genevois se } \\
\text { retrairent par les forteresses du pais, où ilz } \\
\text { estoient commis à garder. } \\
\text { En ce temps ardirent et essillerent les Anglois } \\
\text { grant partie de Bretaigne,et alerent assegièr } \\
\text { Vennes o l'aide des gens du conte de Mont- } \\
\text { fort. Et Robert d'Artois s'en ala en Angle- } \\
\text { terre, où il mourut et fut enterrez à Cantorbie. } \\
\text { Aprés la mort de Robert d'Artois, assembla le } \\
\text { roy d'Angleterre ses hommes... }\end{array}$ \\
\hline $\begin{array}{l}\text { JLB, II, p. 13-17 } \\
\text { Et le roy d'Angleterre en fut durement cou- } \\
\text { roussé, et dist qu'il n'attendroit jamais à } \\
\text { aultre chose tant qu'il eut vengié la mort } \\
\text { dudit messire Robert et mis le pays de Bre- } \\
\text { taigne si au dessoubs, que dedens XL ans ne } \\
\text { seroit recouvré. Et manda tantost lettres par } \\
\text { tout son pays, que nobles et non nobles, tous } \\
\text { fussent appareilliez pour aler avecques luy } \\
\text { au chief du moys exillier et gaster le pays de } \\
\text { Bretaigne. Si fist aprester vaisseaulx et pour- } \\
\text { veances nécessaires. } \\
\text { Comment le roy Edowart vint en Bretaigne } \\
\text { et y assiega III citez en ung jour. Au chief du } \\
\text { moys, le roy Edowart à toute sa compaignie se } \\
\text { mit en mer et vint prendre port assez prez de } \\
\text { la cité de Vennes, là où mesmement messire } \\
\text { Robert d'Artoys et sa compaignie l'avoient } \\
\text { pris l'aultre foys, puis descendi à terre et } \\
\text { assiega la citéde Vennes plus estroitement que } \\
\text { devant, car ily avoit plus grand poissance. } \\
\text { Or estoit la ditte cité merveilleusement ren- } \\
\text { forcée et regarnie et de pourveances et de } \\
\text { gens d'armes, et le pays d'autour estoit si } \\
\text { ouniement gasté, que si grand ost longue- } \\
\text { ment n'y pouoit estre soustenu; si convint } \\
\text { avoir advis sur ce. }\end{array}$ & $\begin{array}{l}\text { CN, p. } 56-58 \\
\text { Aprés la mort de Robert d'Artois, assembla } \\
\text { le roy d'Angleterre ses hommes, tant comme } \\
\text { il peut de son royaume, et des soudoiers } \\
\text { d'Alemaigne et d'ailleurs, et s'en ala par } \\
\text { mer en Bretaigne pour ses hommes secourre. } \\
\text { Et arriva au port delez Vennes et assega la } \\
\text { cité et la fist assaillir pluseurs fois, mais riens } \\
n \text { 'i fist. } \\
\text { Adont envoia le rois Edouart une partie de } \\
\text { ses gens pour assegier la cité de Nantes, et } \\
\text { les conduisoit le conte de Glossestre, le conte } \\
\text { de Guérie, le marquis de Blanquemont et le } \\
\text { conte de Cleves et bien menèrent devant la } \\
\text { cité XX mil hommes. Et adont ala le roy } \\
\text { Edouart devant Rennes en Bretaigne, où } \\
\text { sa gent estoit assegié; et mena moût grant } \\
\text { foison de gent o lui, et avecques lui ala le } \\
\text { prince de Gales, le conte de Hantonne, le } \\
\text { conte de. Derby le conte de Varvic, le conte } \\
\text { d'Arondel et pluseurs autres. Et laissa le } \\
\text { conté de Norhantonne et le conte de Vicestre, } \\
\text { l'evesqué de Nicole, l'evesque de Dureme à } \\
\text { siège devant Venncs. Par ces trois citez qui } \\
\text { furent assegiées et par ces trois ostz, fut le } \\
\text { pais essillié en pluseurs parties. }\end{array}$ \\
\hline
\end{tabular}




\begin{tabular}{|c|c|}
\hline Chronique de Jean Le Bel & Chronique normande \\
\hline 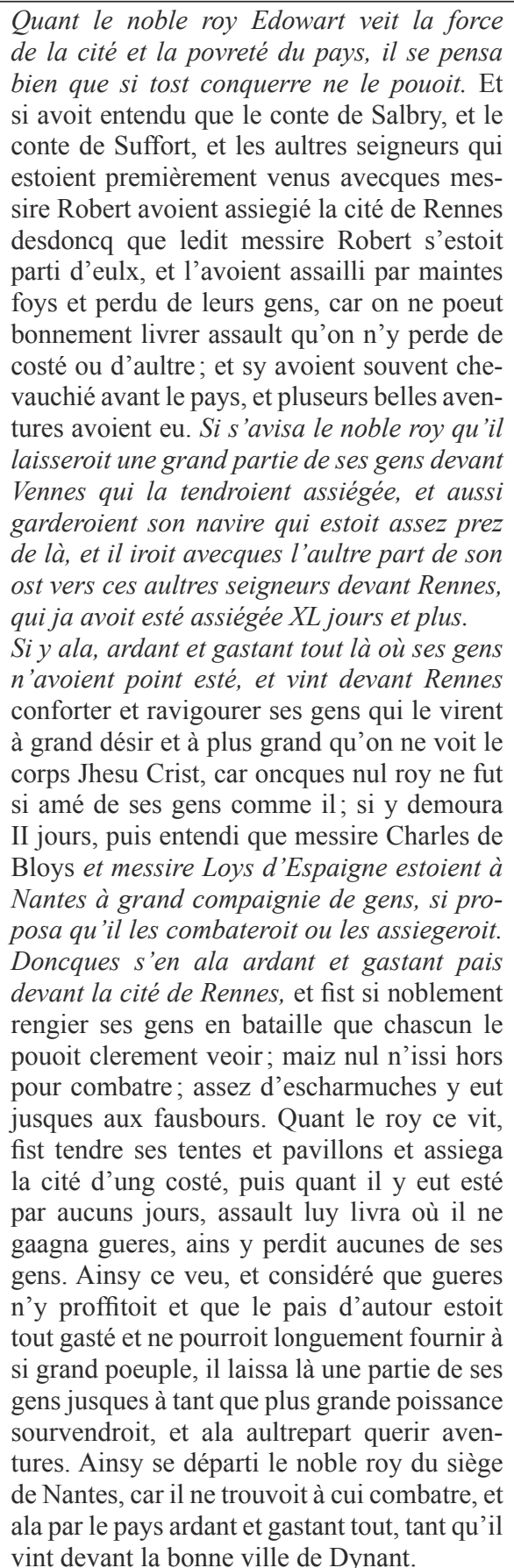 & \\
\hline
\end{tabular}




\begin{tabular}{|c|c|}
\hline Chronique de Jean Le Bel & Chronique normande \\
\hline $\begin{array}{l}\text { Ainsy ardy et gasta le noble roy Edowart } \\
\text { le pays de Bretaigne, et tout à ung jour } \\
\text { assiega à trois osts III cités et une bonne } \\
\text { ville; et quant il eut esté III jours devant } \\
\text { Dynant, et il vit qu'elle n'estoit fermée que } \\
\text { de palys et de fossez, il le fist assaillir, et dura } \\
\text { l'assault longuement, car ceulx de dedens se } \\
\text { deffendoient poissaument. }\end{array}$ & \\
\hline $\begin{array}{l}\text { JLB, II, p. 20-22, 23-25 } \\
\text { Le roy Philippe de France sceut ces nouvelles } \\
\text { que le roy d'Angleterre estoit nouvellement } \\
\text { entré en Bretaigne et ardoit et gastoit tout le } \\
\text { pays, car messire Charles de Bloys luy avoit } \\
\text { mandé et fait assavoir qu'il perderoit tout le } \\
\text { pays s'il n'estoit secouru. Si eut plus grande } \\
\text { paour ledit roy que le roy d'Angleterre } \\
\text { n'entrast par là en France, que son nepveu } \\
\text { messire Charles ne perdist sa terre, la duchié } \\
\text { de Bretaigne. Toutesfois il commanda à son } \\
\text { filz le duc de Normendye qu'il s'aprestast } \\
\text { d'aller celle part, et secourut le duc de } \\
\text { Bretaigne, et gardast bien l'entrée de France, } \\
\text { et luy promit qu'il luy envoieroit tant de gens } \\
\text { aprez luy qu'il luy soufïroit. } \\
\text { Et fist tantost mander par tout son pays que } \\
\text { tous nobles et non nobles fussent tantost } \\
\text { apareilliez pour aller avecques son filz en } \\
\text { Bretaigne. Grande quantité en y ala de nobles } \\
\text { et non nobles, tant que les champs et chemins } \\
\text { estoient tous plains de gens. Quant le duc } \\
\text { de Normendie fut à Angiers, il séjourna là } \\
\text { avecques les aultres seigneurs pour attendre } \\
\text { tandis les venans, et envoya à Nantes vers } \\
\text { messire Charles pour sçavoir de l'estat du } \\
\text { pays et du gouvernement des anemis. }\end{array}$ & $\begin{array}{l}\text { CN p. } 57-58 \\
\text { En l'an de grâce mil CCCXLII, assembla le } \\
\text { roy Phelippe son ost pour aler en Bretaigne } \\
\text { contre le roy Edouart, et envoia devant } \\
\text { Jehan son filz, le roy de Navarre, le conte } \\
\text { d'Alençon, le conte dé Valois, le conte } \\
\text { de Harecourt et plusieurs autres princes, } \\
\text { et furent bien XL mil, hommes, et alerent } \\
\text { premier secourre la cité de Nantes. Quant les } \\
\text { François furent prés, ilz cuiderent l'endemain } \\
\text { avoir la bataille, mais aucuns des bourgois } \\
\text { de Nantes cuiderent en celle nuit livrer la } \\
\text { cité aux Anglois. Mais Miles de Noiers et } \\
\text { plusieurs autres, qui en celle nuit faisoient le } \\
\text { gait, les encontrerent et les prindrent et à tout } \\
\text { leurs requeste les menerent au duc Jehàn de } \\
\text { l'ost de France. } \\
\text { Quant les Anglois sceurent celle aventure, ils. } \\
\text { se deslogerent et s'en alerent à Raines, où le } \\
\text { roy Edouart estoit logié. Et l'endemain lé duc } \\
\text { de Normandie entra en Nantes, et fist couper } \\
\text { le chef à XXX bourgeois, qui furent prins là } \\
\text { nuit en alant en l'ost des Anglois. } \\
\text { Après ce, le duc de Normandie parti de } \\
\text { Nantes à tout son ost et s'en ala vers Rennes. } \\
\text { Et quant Edouart le sceut, il se desloga et s'en } \\
\text { ala devant Vennes, où il avoit de ses gens. Et là } \\
\text { le duc de Normandie le poursuy et fist logier } \\
\text { son ost à deux lieues prés du roy Edouart. } \\
\text { En l'ost du duc. Jehan, estoit Olliviers } \\
\text { de Clicon, Gaudeffroy de Harrecourt et } \\
\text { pluseurs autres chevaliers devant nommez, } \\
\text { qui par trahison s'estoient aliez au conte } \\
\text { de Montfort, et aussi se alierent.il au roy } \\
\text { Edouart par dons et par promesses. Et fut } \\
\text { faite une lettre d'aliance, où li pluseurs } \\
\text { mistrent leurs seaulz, et celle lettre garda } \\
\text { le conte de Salbery de par le roy Edouart. }\end{array}$ \\
\hline
\end{tabular}




\begin{tabular}{|c|c|}
\hline Chronique de Jean Le Bel & Chronique normande \\
\hline 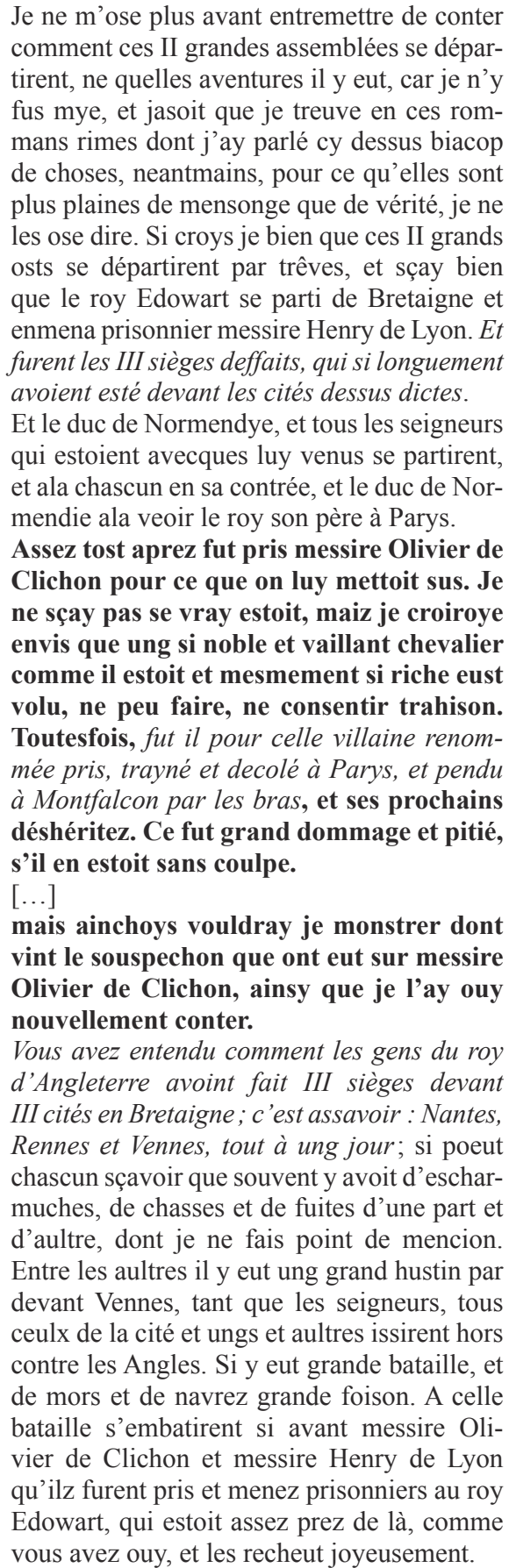 & $\begin{array}{l}\text { Le roy Edouart, par le secret mandement } \\
\text { de Ollivier et de Godeffroy, manda } \\
\text { bataille au duc de Normandie, et en la } \\
\text { fiance des devant nommez, afin que il le } \\
\text { peut desconfire avant que le roy son père } \\
\text { venist.au secours : Et fut la journée dé } \\
\text { la bataille prise au jeudi d'après la Saint } \\
\text { Martin d'iver, en l'an mil CCCXLII. Mais } \\
\text { le roy Phelippe se hasta tant que il vint à } \\
\text { mout grant gens en l'ost son filz devant la } \\
\text { journée, qui estoit accordée dé combatre et } \\
\text { jurée par seremens. Le roy Phelippe et son } \\
\text { filz furent ce jour sur le champ à tout leurs } \\
\text { batailles ordonnées, et au vespre repérèrent } \\
\text { à leurstentes. En ce point furent ces deux } \\
\text { ostz cinq jours sans combatre. Et en ce temps } \\
\text { un cardinal fut envoyé de par le Saint-Père } \\
\text { et traita, tant que trieves furent données } \\
\text { trois ans. Et après ces trieves données, le } \\
\text { roy Edouart s'en ala à toutes ses gens en } \\
\text { Angleterre, et le roy Phelippe s'en alla en } \\
\text { France et laissa Charles de Blois duc et } \\
\text { seigneur de toute Bretaigne Galot. Mais } \\
\text { en Bretaigne Bretonnant avoit pluseurs } \\
\text { forteresses, qui se.tenoient avec le conte de } \\
\text { Montfort et Jehan son filz. } \\
\text { Après ce que le roy de France. et le roy } \\
\text { d'Angleterre furent retraiz en leurs pais, } \\
\underline{\text { le conte de Salbery sceut par sa femme }} \\
\text { que elle avoit esté par force violée du roy } \\
\text { d'Angleterre. Le conte fist son atrait et manda } \\
\underline{\text { ses amis et s'en ala à la court du roy Edouart }} \\
\text { devant les pars et se dessaisi de sa terre par } \\
\text { tele manière que sa femme y prendroit son } \\
\underline{\text { douaire sa vie durant. Dont se parti le conte }} \\
\underline{\text { de Salbery de la court et envoya deffier le }} \\
\text { roy Edouart, et passa la mer et vint au roy } \\
\text { Phelippe et lui bailla les lettres de l'aliance, } \\
\text { que Oliviers, de Clicon et Godeffroy de } \\
\text { Harecourt avoient fait au roy Edouart. Et } \\
\text { moût tost après se parti le conte de-la court } \\
\text { au roy Phelippe, et puis ce temps ne fut veu } \\
\text { en France ne en Angleterre. } \\
\text { [...] }\end{array}$ \\
\hline
\end{tabular}




\begin{tabular}{|c|c|}
\hline Chronique de Jean Le Bel & Chronique normande \\
\hline $\begin{array}{l}\text { Quant ceulx de la cité virent leurs seigneurs } \\
\text { et leurs cappitaines pris, ilz s'en retournèrent } \\
\text { au mielx qu'ilz poeurent en leur cité. Ung } \\
\text { vaillant chevalier qu'on nommoit le baron de } \\
\text { Staffort s'embasti si avant au rechasser qu'il } \\
\text { fut navré et pris et mené en la cité. Quant } \\
\text { ainsy fut avenu, il fut traittié et acordé que } \\
\text { le baron de Staffort, prisonnier, seroit délivré } \\
\text { pour l'ung de ces seigneurs, ou pour messire } \\
\text { Olivier, ou pour messire Henry, lequel que } \\
\text { le roy vouldroit. Ledit roy eut plus chier à } \\
\text { délivrer messire Olivier que messire Henry, } \\
\text { et luy avoit aussy fait tousjours plus de } \\
\text { honnour que audit messire H[enry], car par } \\
\text { aventure ledit messire luy avoit esté plus } \\
\text { contraire et à la vaillant contesse de Montfort } \\
\text { que nul aultre, pour aultre cause non; siques } \\
\text { par cel avantage que le roy fist à messire } \\
\text { Olivier de Clichon et non à messire Henry, } \\
\text { les envieux se penserrent d'aultre chose } \\
\text { que de vérité par aventure, et en sourdi } \\
\text { une souspechon si grande que ledit messire } \\
\text { Olivier villainement en perdit la vye. Et } \\
\text { luy eust mielx valu demourer ou morir en } \\
\text { prison. }\end{array}$ & $\begin{array}{l}\text { Adonc fu pris Olliviérs de Clicon et le fist le } \\
\text { rois décoller à Paris sur I escaffault et fist } \\
\text { le corps pendre à Monfaucon. Et li quiefs } \\
\text { fu portez à Nantes en Bretaigne et fu sur lé } \\
\text { bout, d'un glaive mis sur une des portes de la } \\
\text { cité pour ce que la trahison y fu pourpensée } \\
\text { contre son seigneur. Godeffroy de Harecoùrt } \\
\text { s'en ala par mer au roy Edoùart d'Angleterre, } \\
\text { qui le receut, et le roy le fist bannir par les } \\
\text { quarrefours aux trompes de Paris. Quant les } \\
\text { autres chevaliers seurent celle aventure, ilz } \\
\text { s'assemblèrent et alerent à la dame de Clisson } \\
\text { et lui distrent la mort de son baron. Dont } \\
\text { assembla la dame III cens hommes d'armes } \\
\text { parmy ses traicteurs et s'en alerent au chastel } \\
\text { de Brest... }\end{array}$ \\
\hline
\end{tabular}

Il s'en faut de beaucoup que les textes soient identiques mais le fil du récit est assez largement le même et les similitudes stylistiques, bien réelles, vont au delà d'un simple hasard; cela confirme que l'auteur de la Chronique normande a eu d'une manière ou d'une autre, directe ou indirecte, accès à la chronique de Jean Le Bel et qu'il s'en est inspiré, à côté d'autres sources, pour donner son récit de cet épisode de la guerre de Bretagne.

Si l'on se penche plus précisément sur "l'affaire Salisbury » elle-même, l'on constate par ailleurs que, comme pour les bourgeois de Calais, l'auteur de la Chronique normande ne fait que redonner, certes de manière très rapide, l'ensemble des éléments que contenait le récit de Jean Le Bel. La seule différence concerne le fait que, dans la Chronique normande, c'est le comte de Salisbury qui est allé dénoncer au roi de France, pour se venger d'Édouard III, la trahison d'Olivier de Clisson et des autres barons français alors que Jean Le Bel explique à l'inverse qu'Olivier de Clisson a été victime d'un malentendu : sa libération par le roi d'Angleterre a permis d'insinuer qu'il avait trahi. Mais c'est précisément la possibilité de ce détournement de ce récit de Jean Le Bel qui avait dû rendre particulièrement intéressante l'affaire Salisbury pour l'auteur de la Chronique normande puisque, outre le fait qu'elle salissait la réputation chevaleresque du roi Édouard III, elle lui permettait de démontrer la culpabilité du sire de Clisson.

L'on peut donc affirmer avec certitude que le passage de la Chronique normande et de ses dérivés sur le viol de la comtesse de Salisbury remonte à Jean Le Bel. 